Zbl Arbeitsmed 2019·69:59

https://doi.org/10.1007/s40664-019-0324-y

(c) Springer Medizin Verlag GmbH, ein Teil von Springer Nature 2019

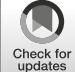

\title{
D. Quarcoo
}

Institut für Arbeitsmedizin, Sozialmedizin und Umweltmedizin, Goethe Universität Frankfurt, Frankfurt am Main, Deutschland

\section{Alles was uns umbringt - eine Reise durch die Welt der Gifte}

\section{Literatur}

wichtiger Bestandteil von Arbeitsmedizin und Umweltmedizin [1-14]. Auch das Zentralblatt für Arbeitsmedizin, Arbeitsschutz und Ergonomie ist seit vielen Jahrzehnten dem Gebiet der Toxikologie verpflichtet.

Aufgrund der zunehmenden Bedeutung von Reisen für die Arbeitswelt und darüber hinaus aufgrund der vielen reisemedizinischen Fragestellungen, die in der täglichen Praxis entstehen, wird das Zentralblatt zukünftig eine neue Serie von Übersichten herausgeben, die sich mit toxikologisch relevanten Zusammenhängen in diesem Sektor befasst.

Es werden u.a. folgende Gebiete behandelt:

- Vergiftungen durch Pilze,

- Vergiftungen durch Schlangenbisse,

- Vergiftungen durch Lebensmittel.

Wie auch bei den anderen Übersichtsserien sind die Autoren der Übersichtsartikel explizit angewiesen, dass sie der interessierten Leserschaft nicht nur Wissen kurz und prägnant übermitteln, sondern auch gezielt Hinweise auf Quellen und Hintergrundinformationen geben sollen.

Gastherausgeber: PD Dr. Dr. David Quarcoo, Facharzt für Arbeitsmedizin, Facharzt für Pädiatrie

\section{Korrespondenzadresse}

\section{PD Dr. Dr. D. Quarcoo}

Institut für Arbeitsmedizin, Sozialmedizin und Umweltmedizin, Goethe Universität Frankfurt Theodor-Stern-Kai 7, 60590 Frankfurt am Main, Deutschland

Quarcoo@em.uni-frankfurt.de

Interessenkonflikt. D. Quarcoo gibt an, dass kein Interessenkonflikt besteht.
1. Sisler JD, Shaffer J, Soo JC, LeBouf RF, Harper M, Qian Y, Lee T (2018) In vitro toxicological evaluation of surgical smoke from human tissue. J Occup Med Toxicol 13:12

2. Ghorbani H, Nezami A, Sheikholeslami B, Hedjazi A, Ahmadimanesh M (2018) Simultaneous measurement of formic acid, methanol and ethanol in vitreous and blood samples of postmortem by headspace GC-FID. J Occup Med Toxicol 13:1

3. Eftekhari S, Aminian O, Moinfar Z, Schettgen T, Kaifie A, Felten M, Kraus T, Esser A (2018) Association of plasma PCB levels and $\mathrm{HbA} 1 \mathrm{c}$ concentration in Iran. J Occup Med Toxicol 13:18

4. Curran J, Cliff R, Sinnen N, Koehle M, Carlsten C (2018) Acute diesel exhaust exposure and postural stability: A controlled crossover experiment. JOccup Med Toxicol 13:2

5. Chai R, Xie H, Zhang J, Ma Z (2018) Sulfur dioxide exposure reduces the quantity of CD19(+) cells and causes nasal epithelial injury in rats. J Occup Med Toxicol 13:22

6. Austigard AD, Svendsen K, Heldal KK (2018) Hydrogen sulphide exposure in waste water treatment. J Occup Med Toxicol 13:10

7. Romaniuk A, Lyndin M, Sikora V, Lyndina $Y$, Romaniuk S, Sikora K (2017) Heavy metals effect on breast cancer progression. J Occup Med Toxicol 12(1):32. https://doi.org/10.1186/s12995-0170178-1

8. Mithander A, Goen T, Felding $G$, Jacobsen $P$ (2017) Assessment of museum staff exposure to arsenic while handling contaminated exhibits by urinalysis of arsenic species. J Occup Med Toxicol 12:26

9. Mastrangelo G, Carta A, Arici C, Pavanello S, Porru S (2017) An etiologic prediction model incorporating biomarkers to predict the bladder cancer risk associated with occupational exposure to aromatic amines: a pilot study. J Occup Med Toxicol 12:23

10. Bengtsson J, Thygesen PS, Kaerlev L, Knudsen LE, Bonde JP (2017) Potential exposure to endocrine disrupting chemicals and selected adverse pregnancy outcomes: A follow-up study of pregnant women referred for occupational counselling. J Occup Med Toxicol 12:6

11. Tay TK, Chan HZ, AhmadTS, Teh KK, Low TH, Wahab NA (2016) Stonefish envenomation of hand with impending compartment syndrome. J Occup Med Toxicol 11:23

12. Ohnishi M, Suzuki M, Yamamoto $M$, Kasai T, Kano H, Senoh H, Higashikubo I, Araki A, Fukushima $S$ (2016) Improved method for measurement of multi-walled carbon nanotubes in rat lung. JOccup Med Toxicol 11:44

13. Mund ME, Gyo C, Bruggmann D, Quarcoo D, Groneberg DA (2016) Acetylsalicylic acid as a potential pediatric health hazard: Legislative aspects concerning accidental intoxications in the European Union. J Occup Med Toxicol 11:32

14. Malara P, Fischer A, Malara B (2016) Selected toxic and essential heavy metals in impacted teeth and the surrounding mandibular bones of people exposed to heavy metals in the environment. JOccup Med Toxicol 11:56 